\title{
The Effect of Exercise Treadmill of Rat Liver Tissue
}

\author{
Murat TAŞ \\ Celal Bayar University, Manisa, Turkey
}

\begin{abstract}
Our study was designed to determine the level of oxidative stress parameters in rats consisting of treadmill exercise performed at different times. The rats in the treadmill exercise groups were subjected to run on a treadmill for 15 and 30 mins. once a day, seven times a week, continued for 28 days. Compliance with the normal distribution of variables was evaluated by Kolmogorov Smirnov Test. Comparisons between groups were analyzed using one-way ANOVA post hoc Least Significant Difference (LSD) Test. $p<0.05$ was considered statistically significant differences. As a result, superoxide dismutase (SOD) $1.09 \pm 0.23,3.95 \pm 2.26$, glutathione (GSH) and malonic dialdehyde (MDA) was determined to be $1.46 \pm 0.41$. Short duration exercise group: $1.22 \pm 0.14$ SOD, GSH $3.12 \pm$ 0.17 , and MDA $1.64 \pm 0.58$. Moderate exercise group: SOD $1.12 \pm 0.19$, GSH $2.49 \pm 0.42$, and MDA $1.16 \pm 0.49$ level was found in. As a result, it is more effective than moderate exercise on antioxidant activity, but the short-term exercise training performed in animals oxidant/antioxidant balance between energy systems said to be linked to the system.
\end{abstract}

Keywords: rat, exercise, liver, free radical

\section{Introduction}

The organism or tissue usually need energy production to adapt to achieve the aerobic pathways. But, it depends on the type of exercise and intensity together, the production of energy needed by the body that results in the production of high levels of reactive oxygen species anaerobic energy production is usually tried to be provided on aerobic way (Hakim et al., 1998).

Free radicals are especially cancer, are indicated as the cause of many diseases, including aging. Aerobic organisms have a protection system enzymatic and non-enzymatic antioxidant properties that work to neutralize free radicals formed when oxygen and other ions originating from various sources (Walther, Gielen, \& Hambrecht, 2004; Bassuk \& Manson, 2003).

The life and integrity of the organism depends on maintaining homeostatic balance. Homeostasis is under constant threat from both internal as well as external factors. Cells against the destructive effects of free radicals and have antioxidant systems of the organism as a whole. These mechanisms tend to act to eliminate the precursor of free oxygen radicals, or free radicals formed by cleaning. They show tissue antioxidant systems differ from each other in terms of capacity. High brain metabolic activity, oxygen consumption in excess tissues, such as liver and kidney, have a higher antioxidant capacity compared to other tissues (Fiçıcılar, Zergeroğlu, Tekin, \& Ersöz, 2003; Rush, Denniss, \& Graham, 2005).

In the study, to explain the quality of life and duration of exercise in terms of efficiency, violent muscle, erythrocyte antioxidant enzyme activities, and their impact on the oxidizing agents, treadmill aimed short and

Murat TAŞ, Dr., associate professor, School of Physical Education and Sports, Celal Bayar University. 
antioxidant enzymes in rats who exercise at moderate to examine activity levels.

\section{Methods}

\section{Animals and Groups}

Fifty-six male rats (12 weeks old, Albino-Wistar), fed with standard laboratory chow and water, were used in the study. Animal experimentations were approved by the Ethical Committee of the Ataturk University and carried out in an ethically proper way by following the guidelines provided.

\section{Experimental Protocol}

Rats in the exercise group were introduced to running on a motor driven rodent treadmill (TME 9805 Treadmill Exerciser, Commat Iletisim, Ankara, Turkey). The treadmill was equipped with an electric shocking grid on the rear barrier to provide exercise motivation to the rats. The rats in the treadmill exercise groups were subjected to run on a treadmill for 15 and 30 mins. once a day, seven times a week, continued for 28 days. Exercise load for the exercise groups consisted of running at a speed of 2 meters/mins. for the first 5 mins., at a speed of 5 meters $/$ mins. for the next 5 mins., and then at a speed of 8 meters $/ \mathrm{mins}$. for the last 20 mins., with the $0^{\circ}$ inclination. This intensity corresponded to the low-intensity treadmill exercise $(\%$ maximal oxygen consumption) according to the rats in this age (Bedford, Tipton, Wilson, Oppliger, \& Gisolfi, 1979).

Biochemical investigation of liver tissues after macroscopic analysis, rat tissues were kept at $86{ }^{\circ} \mathrm{C}$. One hundred mg of tissues from each rat was firstly perfused with PBS/heparin and then homogenized in a specific homogenate tampon (appropriate buffer) on ice by ultra-turrax after grinding in liquid nitrogen. Then, they were centrifuged according to the manufacturer's instructions. Then, for biochemical investigation, superoxide dismutase (SOD) activity, and malonic dialdehyde (MDA) and glutathione (GSH) levels from each supernatant were measured in duplicate with highly sensitive kits (Cayman-706002, USA), and Cell Biolabs-STA-330 and 312 (USA), respectively, specifically designed for rat tissue, according to the manufacturer's instructions. The protein concentrations were determined by the Lowry method using commercial protein standards (Sigma Aldrich, Total protein kit-TP0300-1KT-USA). All the data was presented as the mean seven standard deviation (SD) results based on per mg of protein.

SOD measurement principles. Cayman's Superoxide Dismutase Assay Kit utilizes a tetrazolium salt for detection of superoxide radicals generated by xanthine oxidase and hypoxanthine. One unit of SOD is defined as the amount of enzyme needed to exhibit $50 \%$ dismutation of the superoxide radical. The SOD assay measures all three types of SOD $(\mathrm{Cu} / \mathrm{Zn}, \mathrm{Mn}$, and FeSOD). There are two total sod activity (cytosolic and mitochondrial). To separate the two enzymes, centrifuge supernatant at $10.000 \mathrm{~g}$ for 15 mins. at $4{ }^{\circ} \mathrm{C}$. The resulting $10.000 \mathrm{~g}$ supernatant will contain cytosolic SOD. Samples can be assayed in the absence of Xanthine Oxidase to generate a sample background using Elisa reader the absorbance at $440 \mathrm{~nm}$.

Total GSH measurement principles. The OxiSelect ${ }^{\mathrm{TM}}$ Total Glutathione (GSSG/GSH) Assay Kit is a quantitative assay for measuring the total glutathione content within a sample (GSH/GSSG). Glutathione Reductase reduces oxidized glutathione (GSSG) to reduced glutathione (GSH) in the presence of NADPH. Subsequently, the chromogen reacts with the thiol group of GSH to produce a colored compound that absorbs at $405 \mathrm{~nm}$. The total glutathione content in unknownsamples is determined by comparison with the predetermined glutathione standard curve. The rate of chromophore production is proportional to the 
concentration of glutathione within the sample. The rate can be determined from the absorbance change over time. Metaphosphoric acid is provided to remove interfering proteins or enzymes from samples.

MDA measurement principles. The Thiobarbituric Acid Reactive Substances (TBARS) Assay Kit is a tool for the direct quantitative measurement of MDA in biological samples. The unknown MDA containing samples or MDA standards are first reacted with TBA at $95{ }^{\circ} \mathrm{C}$. After a brief incubation, the samples and standards can be read either spectrophotometrically or fluorometrically. The MDA content in unknown samples is determined by comparison with the predetermined MDA standard curve.

\section{Statistical Analysis}

Statistical analysis Statistic Package for Social Science (SPSS) 18.0 (SPSS Inc., Chicago, IL) was performed using the program. Compliance with the normal distribution of variables was evaluated by KolmogorovSmirnov test. Comparisons between groups were analyzed using one-way ANOVA post hoc Least Significant Difference (LSD) Test. $p<0.05$ was considered statistically significant differences.

\section{Results}

When examining Table 1 of the control group: SOD $1.09 \pm 0.23$, GSH $3.95 \pm 2.26$, and MDA was determined to be $1.46 \pm 0.41$. Short duration exercise group: $1.22 \pm 0.14 \mathrm{SOD}$, GSH $3.12 \pm 0.17$, and MDA $1.64 \pm 0.58$. Moderate Exercise group: SOD $1.12 \pm 0.19$, GSH $2.49 \pm 0.42$, and MDA $1.16 \pm 0.49$ level was found in.

Table 1

Liver of The Study Group, SOD, GSH, MDA Levels

\begin{tabular}{llll}
\hline \multirow{2}{*}{ Groups } & SOD & GSH & MDA \\
\cline { 2 - 4 } & $(U / \mathrm{mg}$ protein) & $(\mathrm{nmol} / \mathrm{mg}$ protein) & (nmol/mg protein) \\
\hline Control & $1.09 \pm 0.23$ & $3.95 \pm 2.26$ & $1.46 \pm 0.41$ \\
Short duration exercise & $1.22 \pm 0.14^{*}$ & $3.12 \pm 0.58$ & $1.64 \pm 0.17$ \\
Moderate exercise & $1.12 \pm 0.19^{*}$ & $2.49 \pm 0.42^{*}$ & $1.16 \pm 0.49^{*}$ \\
\hline
\end{tabular}

Note. * SOD level of the short duration exercise group was higher than control and moderate exercise group, and in the moderate exercise group, both GSH and MDA levels were statistically significant compared to the other groups $(p<0.05)$.

\section{Discussion}

Free radical increase will occur and the increase in long-term aerobic exercise results are neutralized by antioxidant system without damaging the muscle. Protection level of antioxidant enzymes in skeletal chassis has been suggested to be associated with the amount of oxygen used by the tissue. With increasing levels of free radicals in the implementation of the detrimental effects of exercise are well known to be relevant protocol.

Antioxidant usage is a very attractive topic, because excess reactive oxygen species (ROS) is now recognized to be associated with some prevalent diseases, as well as with the normal aging process (Urso \& Clarkson, 2004; Seifried, Anderson, Fisher, \& Milner, 2007; Nelson, Bose, Grunwald, Myhill, \& McCord, 2006)

During normal cellular activities, various metabolic processes inside of cells produce free radicals and ROS. Production of free radicals is increased by physical activity in several ways. Therefore, exercise can cause an imbalance between ROS and antioxidants, referred to as oxidative stress. ROS, when present in a high enough concentration, can affect cellular components, especially lipids, which initiate a chain reaction called lipid peroxidation (Alonso, Collado, \& González-Gallego, 2006; Mikami, Sumida, Ishibashi, \& Ohta, 2004) 
The accumulation of lipid peroxidation products provides the most common biochemical marker of oxidative stress. The TBARS assay is the most widely used for assessment of lipid peroxidation, although it is somewhat controversial due to lack of specificity (Alonso et al., 2006; Kakarla, Vadluri, \& Kesireddy, 2005).

Acute-intense exercise does not elicit the same response as long-term exercise training. Although regular exercise training decreases oxidative stress (Vincent, Morgan, \& Vincent, 2004), strenuous acute physical exercise can cause oxidative stress and subsequent damage to cellular proteins (Gül et al., 2006) lipids and nucleic acids as well as changes to the glutathione system (Burneikoa et al., 2006). Gül et al. (2006) had found the decrease in superoxide dismutase activity in heart during the acute exercise in untrained rats, also studied effect of short-term intensive exercise on the some oxidative parameters (Dayan et al., 2005) Chronic resistance training strengthens the antioxidant defense system (Burneikoa et al., 2006). In a research conducted in rats for eight weeks and five days two weeks the rats who exercise increase in liver tissue SOD, catalase (CAT) values in decline and that the significant increase in the reported levels of serum total oxidant statu (TOS) (Bloomer, Goldfarb, Wideman, McKenzie, \& Consitt, 2005; Liu et al., 2000).

Rats were subjected to 60 mins. of treadmill exercise per $\mathrm{kg}$ antioxidant agent of $1 \mathrm{mg}$ melatonin $30 \mathrm{mins}$. before the muscle has been shown to have melatonin against oxidative damage caused by acute exercise on the potential protective effect (Fıçıcılar, Zergeroğlu, Tekin, \& Ersöz, 2003). Total antioxidant status (TAS) values reported in a study of acute strenuous exercise on horses that they do significantly reduce. Intense exercise stress in rats melatonin and deoxyribonucleic acid (DNA) damage occurs in muscle and ascorbic acid in a study investigating the effects of oxidative stress; The exercise of ascorbic acid to increase the TOS values to reduce it, it is stated that exercise improves design and ascorbic acid. However, melatonin had no effect on the TOS and TAS, both alone exercise is associated with increased DNA damage with melatonin supplements. Muscle degeneration increased with exercise stress, and melatonin has been found to reduce muscle damage induced by oxidative stress induced by exercise stress of ascorbic acid (Fiçıcılar et al., 2003; Parise, Brose, \& Tarnopolsky, 2005; Ağırbaş, 2013)

As a result, it is more effective than moderate exercise on antioxidant activity, but the short-term exercise training performed in animals oxidant/antioxidant balance between energy systems said to be linked to the system.

\section{Conclusion}

In summary, we can say that excessive exercise increases oxidative stress and antioxidant capacity decreases. Antioxidant supplements to people do exercise for increasing the capacity of the reduction of oxidative stress and antioxidant may be useful. Taking antioxidant supplements may increase the performance of that exercise.

\section{References}

Ağırbaş, Ö. (2013). The effects of melatonin and ascorbic acid on muscular DNA damage and oxidative stress in intensive exercise stressed rats. Institute of Health Sciences Department of Sports and Health Sciences (Ph.D.), Atatürk University.

Alonso, M., Collado, P. S., \& González-Gallego. (2006). Melatonin inhibits the expression of the inducible isoform of nitric oxide synthase and nuclear factor kappa B activation in rat skeletal muscle. Journal of Pineal Research, 41, 8-14.

Bassuk, S. S., \& Manson, J. F. (2003) Physical activity and the prevention of cardiovascular disease. Current Atherosclerosis Reports, 5, 299-307.

Bedford, T. G., Tipton, C. M., Wilson, N. C., Oppliger, R. A., \& Gisolfi, C. V. (1979). Maximum oxygen consumption of rats and its changes with various experimental procedures. Journal of Applied Physiology, 47, 1278-1283. 
Bloomer, R. J., Goldfarb, A. H., Wideman, L., McKenzie, M. J., \& Consitt, L. A. (2005). Effects of acute aerobic and anaerobic exercise on blood markers of oxidative stress. Journal of Strength Condition Research, 19, 276-285.

Burneikoa, R. C. M., Diniz, Y. S., Galhardib, C. M., Rodriguesb, H. G., Ebaida, G. M. X., Fainea, L. A., ... Novellia, E. L. B. (2006). Interaction of hypercaloric diet and physical exercise on lipid profile, oxidative stress and antioxidant defenses. Food and Chemical Toxicology, 44, 1167-1172.

Dayan, A., Rotstein, A., Pinchuk, I., Vodovicz, A., Lencovski, Z., Lichtenberg, D., O, Inbar. (2005). Effect of a short-term graded exhaustive exercise on the susceptibility of serum lipids to oxidation. Int. J. Sports Med, 26, 732-738.

Fıçıcılar, H., Zergeroğlu, A. M., Tekin, D., Ersöz, G. (2003). The effects of acute exercise on plasma antioxidant status and platelet response. Thrombosis Research, 111, 267-271.

Gul, M., Demircan, B., Taysi, S., Oztasan, N., Gumustekin, K., Siktar, ... Dane, S. (2006). Effects of endurance training and acute exhaustive exercise on antioxidant defense mechanisms in rat heart. Comp. Biochem Physiol, 143, 239-245.

Hakim, A. A., Petrovitch, H., Burchfiel, C. M., Ross, G. W., Rodriguez, B. L., White, L. R., ...Abbott, R. D. (1998). Effects of walking on mortality among nonsmoking retired men. N. Eng. J. Med, 338, 94-99.

Kakarla P, Vadluri G, \& Kesireddy R. S. (2005). Response of hepatic antioxidant system to exercise training in aging female rat. Journal of Experimental Zoology, 303(3), 203-208.

Liu, J., Yeo, H. C., Övervik-Douki, E., Hagen, T., Doniger, S. J., Chu, D. W., ... Ames, B. N. (2000). Chronically and acutely exercised rats: biomarkers of oxidative stress and endogenous antioxidants. J. Appl. Physiol, 89, 21-8.

Mikami, T., Sumida, S., Ishibashi, Y., \& Ohta, S. (2004). Endurance exercise training inhibits activity of plasma GOT and liver caspase-3 of mice (correction of rats) exposed to stress by induction of heat shock protein 70. Journal of Applied Physiology, 96, 1776-1781.

Nelson, S. K, Bose, S. K, Grunwald, G. K, Myhill, P., \& McCord, J. M. (2006). The induction of human superoxide dismutase and catalase in vivo: a fundamentally new approach to antioxidant therapy. Free Radic. Biol. Med, 40, 341-347.

Parise, G., Brose, A. N., \& Tarnopolsky, M. A. (2005). Resistance exercise training decreases oxidative damage to DNA and increases cytochrome oxidase activity in older adults. Exp. Gerontol, 40, 173-180.

Rush, J. W., Denniss, S. G., \& Graham, D. A. (2005). Vascular nitric oxide and the oxidative stress: determinants of endothelial adaptations to cardiovascular disease and to physical activity. Can. J. Appl. Physiol, 30, 442-474.

Seifried, H. E., Anderson, D. E., Fisher, E. I., \& Milner, J. A. (2007). A review of the interaction among dietary antioxidants and reactive oxygen species. $J$. Nutr. Biochem, 18, 567-579.

Urso, M. L., \& Clarkson, P. M. (2003). Oxidative stress, exercise, and antioxidant supplementation. Toxicology, 189, 41-54.

Vincent, H. K., Morgan, J. W., \& Vincent, K. R. (2004). Obesity exacerbates oxidative stress levels after acute exercise. Med Sci. Sports Exerc., 36, 772-779.

Walther, C., Gielen, S., \& Hambrecht, R. (2004). The effect of exercise training on endothelial function in cardiovascular disease in humans. Exerc. Sport Sci. Rev., 32, 129-134. 